Copyright (C) 2013 IEEE. Personal use of this material is permitted. Permission from IEEE must be obtained for all other uses, in any current or future media, including reprinting/republishing this material for advertising or promotional purposes, creating new collective works, for resale or redistribution to servers or lists, or reuse of any copyrighted component of this work in other works. 


\title{
Low-Complexity Instantaneous Ambiguity Resolution with the Affine-constrained GNSS \\ Attitude Model
}

\author{
Gabriele Giorgi, and Peter J.G. Teunissen.
}

\begin{abstract}
In this contribution a new approach to ambiguity resolution for Global Navigation Satellite System-based attitude estimation applications is discussed and tested. This approach enhances the observation model by means of additional linear constraints. These constraints are implicitly derived by applying an affine transformation to the unknown realvalued parameters, and largely aid the integer ambiguity estimation through an improved float estimation. The new method is characterized by a lower computational complexity than a rigorous nonlinearly-constrained GNSS attitude model, and at the same time it guarantees higher performance than unconstrained, and therefore weaker, models. The ambiguity resolution performance of the affine-constrained approach is tested through a series of simulated as well as actual data-sets, demonstrating the validity and usefulness of the proposed approach for multi-antenna GNSS-based attitude determination applications.
\end{abstract}

Index Terms

GNSS Attitude Determination - Carrier Phase Ambiguity Resolution - Multivariate Integer Least Squares Orthonormality-Constrained Attitude Model - Affine-Constrained Attitude Model.

\section{INTRODUCTION}

GNSS-based attitude determination is a viable alternative to methods based on different sensing equipment, such as gyroscopes or star-trackers. Although the accuracy of a stand-alone GNSS attitude system might not be comparable with the one provided by other modern attitude sensors, a GNSS-based system presents several advantages. It is inherently driftless, a GNSS receiver has low power consumption, it requires minor maintenance, and it is not as expensive as other high-precision systems, e.g., laser gyroscopes [1], [2], [3], [4], [5], [6], [7], [8], [9], [10], [11],

Gabriele Giorgi is a researcher at the Institute for Communications and Navigation, Technische Universität München, in Theresienstrasse 90 , Room N4406, 80333 Munich, Germany. E-mail: gabriele.giorgi@tum.de.

Peter J.G. Teunissen is a Federation Fellow of the Australian Research Council (ARC), professor of geodesy and navigation and leader of Curtin University of Technologys Global Navigation Satellite System (GNSS) research lab, in Bentley, 6845 Perth, Western Australia. E-mail: p.teunissen@curtin.edu.au

Manuscript received September 28, 2011; revised July 16, 2012. 
[12], [13], [14], [15], [16], [17].

In order to achieve highly precise attitude estimations, GNSS carrier phase data are employed, whose measurement noise is two orders of magnitude lower than the code (pseudorange) measurement error. However, each carrier phase reading is ambiguous by an unknown number of whole cycles, since only the fractional part of the locked GNSS signal is detected by the tracking loop. The process of resolving this unknown to its correct integer value for each tracked GNSS signal goes under the name of ambiguity resolution. Several methods have been discussed in literature for the purpose of ambiguity resolution, falling in different classification categories. A first category includes those methods that take advantage of the time-varying relative receiver-to-satellite geometry to correctly estimate the integer ambiguities. These motion-based methods inherently require some time in order to be effective, and thus do not provide instantaneous solutions. A second category is the one of search-based methods. These methods extensively search for the unknown ambiguities by evaluating admissible sets of potential integer-valued candidates. Thus search-based methods can in principle be used for instantaneous ambiguity resolution, since the presence of motion is not required. The method presented in this work belongs to the class of search-based methods. Further means of classification are given in [11], [18], [19], and references therein.

GNSS-based attitude applications usually can benefit from additional information: the antennas relative geometry has to be precisely known in order to estimate the orientation of the platform. If correctly accounted for, this information can be used to strengthen the functional modeling and to achieve very high performance in terms of reliable ambiguity estimation. A rigorous modeling of the GNSS-based attitude problem is given in [20], where the observation model is formulated in terms of the unknown integer ambiguities and an unknown orthonormal rotation matrix. This model, hereafter referred to as the Orthonormality-Constrained Attitude Model (OC-AM), includes the whole set of nonlinear geometrical constraints given by the a-priori knowledge of the local antenna coordinates, i.e., the positions of the antennas in a frame integral with the platform are assumed completely known, and the global baseline coordinates can be derived by means of a rigid rotation. This approach yields very high ambiguity resolution performance, as demonstrated in [9], at the cost of having an inherently complex search algorithm due to the nonlinear nature of the given constraints.

Recently, an alternative functional model was introduced [21]: the Affine-Constrained Attitude Model (AC-AM). By disregarding the orthonormality of the rotation matrix, the baseline coordinates are re-parameterized by means of an affine transformation. Under certain conditions that will be stated, this transformation yields linear constraints that aid the ambiguity estimation process. The AC-AM is weaker than its orthonormality-constrained counterpart, but at the same time it guarantees higher performance than what is achieved by applying unconstrained models, in which the geometrical information is completely disregarded.

The new AC-AM is particularly apt for multi-antenna ambiguity resolution, as its strength generally increases with the number of baselines employed. It is the objective of this work to present empirical evidence of the performance of the new affine-constrained ambiguity resolution method. 
The structure of this contribution is as follows. Section II introduces the models that will be discussed and compared: the unconstrained, the orthonormality-constrained and the affine-constrained formulations of the GNSS-based attitude model. Section III addresses the multivariate integer least-squares (ILS) solution of each model, highlighting the different properties that characterize each formulation. Finally, section IV presents experimental results obtained by applying the discussed ambiguity resolution methods to simulated and actual GNSS observations data sets.

\section{The GNSS ATtitude Models}

\section{A. The Unconstrained Model}

Consider a platform equipped with $r+1$ GNSS antennas. If these simultaneously track the same $s+1$ GNSS satellites on the same $f$ frequencies, the following linear(ized) system of double-differenced (DD) observation equations for the set of $2 f s r$ code and carrier phase DD measurements can be formulated [20][22],

$$
\begin{gathered}
\mathrm{E}(Y)=M X+N Z ; \quad \mathrm{D}(\operatorname{vec}(Y))=Q_{Y Y} \\
Z \in \mathbb{Z}^{f s \times r} ; X \in \mathbb{R}^{3 \times r}
\end{gathered}
$$

with $\mathrm{E}($.$) and \mathrm{D}($.$) the expectation and dispersion operators, respectively, and where Y$ is the $2 f s \times r$ matrix whose columns contain the DD code and phase observations from each baseline, $X$ is the $3 \times r$ matrix of real-valued baseline coordinates, $Z$ is the $f s \times r$ matrix of integer ambiguities, $M$ is the $2 f s \times 3$ matrix of DD unit line-ofsight vectors, and $N$ is the $2 f s \times f s$ matrix containing the carrier wavelengths. Under the assumption of short baseline separations between antennas - which is a valid assumption for attitude determination applications, since the dimensions of platforms rarely exceed hundreds of meters - the deviations between the different line-of-sight vectors from each antenna are discarded, and hence the same line-of-sight vectors are used in matrix $M$. Also, under the same assumption of short-baselines, the atmospheric offsets are eliminated by the differencing operations. In order to define the variance-covariance (v-c) matrix of the matrix of observables $Y$, the vec operator is applied. This stacks the columns of the $2 f s \times r$ matrix $Y$ into the vector vec(Y) of order $2 f s r$, whose dispersion is then described by the $2 f s r \times 2 f s r$ v-c matrix $Q_{Y Y}$.

In the following, it will be assumed that the baselines are formed by differencing with respect a common main antenna. Thus, the v-c matrix $Q_{Y Y}$ can be expressed as

$$
Q_{Y Y}=P \otimes Q_{y y}
$$

with $Q_{y y}$ the v-c matrix that describes the dispersion of each set of baseline observations, and $P$ the $r \times r$ matrix that introduces correlation between baseline observations due to the common antenna used to form the DD observations. For the properties of the vec operator and the Kronecker product, we refer to e.g. [23], [24]. With a set of $r$ baselines

formed as illustrated in Figure 1, matrix $P$ is obtained as $P=\frac{1}{2}\left(I_{r}+e_{r} e_{r}^{T}\right)$, with $e_{r}$ an order- $r$ vector of ones. Further details regarding model (1) are given in [21].

Note that model (1) linearly relates the observation matrix $Y$ to the unknown matrix parameters $X$ and $Z$, and 


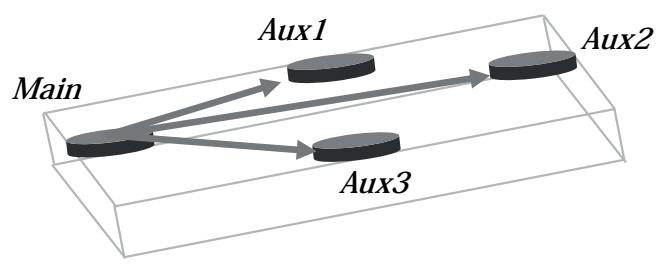

Fig. 1. Baselines are formed by differencing with respect to the same Main antenna.

that the only matrix constraint is the one imposed on the ambiguity matrix $Z$, whose entries are integer-valued. Therefore, model (1) will be referred to as the Unconstrained Attitude Model (UC-AM).

\section{B. Attitude Determination}

The attitude of a rigid body (or platform) is described by the orientation of its body frame with respect to a reference frame. Let the body frame baseline coordinates of the $r+1$ GNSS antennas be collected in the $3 \times r$ matrix $B$ and let the corresponding, GNSS determined, reference frame coordinates of these baselines be collected in the $3 \times r$ matrix $X$. Then the two matrices $X$ and $B$ are related as

$$
X=R B \quad ; \quad X \in \mathbb{R}^{3 \times r}, B \in \mathbb{R}^{3 \times r}, R \in \mathbb{O}^{3 \times 3}
$$

with $(\mathbb{O}$ denoting the class of orthonormal matrices. The orthonormality constraints imply that the columns of $R$ are all of lenght one (normality) and that each scalar product between any two of its columns is zero (orthogonality). Relation (3) can be used to estimate the attitude matrix $R$ from given $X$ and $B$. In case of GNSS attitude determination, $B$ is assumed known, while $X$ is estimated from GNSS data. For example, following the solution of model (1), a precise matrix estimate $\check{X}$ of $X$ becomes available, and $R$ can be least-squares estimated as

$$
\check{R}=\arg \min _{R \in \mathbb{O}^{3 \times 3}}\|\operatorname{vec}(\check{X}-R B)\|_{Q}^{2}
$$

where the weighted squared norm reads $\|\cdot\|_{Q}^{2}=(\cdot)^{T} Q^{-1}(\cdot)$. This nonlinear least-squares problem can be solved by means of iterative descent methods, like e.g. the Newton method. If $Q$ turns out to be diagonal, or may be approximated as such, then very fast methods have been devised to find the minimization point, as shown in [25]. An overview of available means of solving (4) is given in [26].

However, not every $3 \times r$ body frame baseline matrix $B$ can be used to estimate a $3 \times 3$ rotation matrix. For example, configurations of $r$ baselines obtained by lining up $r+1$ antennas cannot be used to estimate the complete platform attitude. One rotation angle would remain ambiguous, since it would not be possible to determine the rotation about the antennas alignment direction. This limitation can be formalized as in [21]: a full (3×3) rotation matrix $R$ cannot be estimated if the span of the baselines is not at maximum, i.e., if $q=\operatorname{rank}(B)<\min (3, r)$. Therefore, we generalize relation (3) to

$$
X=R B \quad ; \quad X \in \mathbb{R}^{3 \times r}, B \in \mathbb{R}^{q \times r}, R \in \mathbb{O}^{3 \times q}
$$


The minimization problem (4) is then generalized accordingly by replacing the constraints $R \in \mathbb{O}^{3 \times 3}$ by $R \in \mathbb{O}^{3 \times q}$.

\section{The Orthonormality- and Affine-Constrained Models}

Attitude determination based on the unconstrained model (1) implies that relation (5) is only used after one has solved for the baseline matrix $X$. Hence, such baseline solution will not benefit from the known body frame geometry of the antenna-configuration. An alternative approach is to make use of relation (5) from the start. By substituting (5) into (1), we obtain the model

$$
\begin{gathered}
\mathrm{E}(Y)=M R B+N Z ; \quad \mathrm{D}(\operatorname{vec}(Y))=Q_{Y Y} \\
Z \in \mathbb{Z}^{f s \times r} ; R \in \mathbb{O}^{3 \times q}
\end{gathered}
$$

This is the Orthonormality-Constrained Attitude Model (OC-AM) as introduced in [20], see also [27], [9]. It has the body frame antenna-geometry incorporated into the system from the start. The model is now parametrized in the orthonormal attitude matrix $R \in \mathbb{O}^{3 \times q}$, instead of in the baseline matrix $X \in \mathbb{R}^{3 \times r}$. The incorporation of these orthonormality constraints results in a stronger model and therefore also in improved ambiguity resolution performance. However, since the orthonormality-constraints are nonlinear, the ambiguity objective function increases significantly in complexity (i.e. the ambiguity search space is not ellipsoidal anymore, see e.g. [28]) and the search for its integer minimizer requires a higher computational load.

A model of intermediate strength is obtained if one disregards the orthonormality constraints,

$$
\begin{gathered}
\mathrm{E}(Y)=M R B+N Z ; \quad \mathrm{D}(\operatorname{vec}(Y))=Q_{Y Y} \\
Z \in \mathbb{Z}^{f s \times r} ; R \in \mathbb{R}^{3 \times q}
\end{gathered}
$$

This is the Affine-Constrained Attitude Model (AC-AM). It is a linear model that avoids the complexity of OC-AM. By replacing the nonlinear constraints $R \in \mathbb{O}^{3 \times q}$ with $R \in \mathbb{R}^{3 \times q}$, one effectively assumes that $X$ and $B$ are related through the affine transformation $X=R B$. This relation imposes a set of $r-q$ linear constraints if $r>q$, i.e. if the number of baselines is larger than their span. In [21] the ILS-solution of the AC-AM is developed with the affine constraints given in implicit as well as explicit form.

\section{Multivariate Integer Least-SQuares Theory}

\section{A. Three Different Minimizers}

Each of the models (1), (6) and (7) can be solved by making use of the principles of multivariate integer leastsquares estimation [20], [21], [29]. The three ILS minimization problems that we will address in this contribution are therefore the ones for the UC-AM model (1),

$$
\left\{\begin{array}{c}
\check{Z}_{\mathrm{uc}} \\
\check{X}_{\mathrm{uc}}
\end{array}\right\}=\arg \min _{Z \in \mathbb{Z}^{f} \times r \times, X \in \mathbb{R}^{3 \times r}}\|\operatorname{vec}(Y-M X-N Z)\|_{P \otimes Q_{y y}}^{2}
$$


the AC-AM model (7),

$$
\left\{\begin{array}{l}
\check{Z}_{\mathrm{ac}} \\
\check{R}_{\mathrm{ac}}
\end{array}\right\}=\arg \min _{Z \in \mathbb{Z}^{f s \times r}, R \in \mathbb{R}^{3 \times q}}\|\operatorname{vec}(Y-M R B-N Z)\|_{P \otimes Q_{y y}}^{2}
$$

and the OC-AM model (6),

$$
\left\{\begin{array}{l}
\check{Z}_{\mathrm{oc}} \\
\check{R}_{\mathrm{oc}}
\end{array}\right\}=\arg \min _{Z \in \mathbb{Z}^{f s \times r}, R \in \mathbb{O}^{3 \times q}}\|\operatorname{vec}(Y-M R B-N Z)\|_{P \otimes Q_{y y}}^{2}
$$

Since one can order the strength of the three attitude models as $\mathrm{UC} \leq \mathrm{AC} \leq \mathrm{OC}$, the probabilities of correct integer estimation (or ambiguity success-rates) of the three integer ambiguity estimators can be ordered as

$$
\mathrm{P}\left(\check{Z}_{\mathrm{uc}}=Z\right) \leq \mathrm{P}\left(\check{Z}_{\mathrm{ac}}=Z\right) \leq \mathrm{P}\left(\check{Z}_{\mathrm{oc}}=Z\right)
$$

In the following sections we will show by how much one can expect these success-rates to differ. First, however, we give the intermediate steps for computing the three different ambiguity objective functions.

\section{B. The Unconstrained Float Solution}

Without the integer constraints $Z \in \mathbb{Z}^{f s \times r}$, the minimization problem (8) results in the so-called float solution

$$
\left\{\begin{array}{l}
\hat{Z}_{\mathrm{uc}} \\
\hat{X}_{\mathrm{uc}}
\end{array}\right\}=\arg \min _{Z \in \mathbb{R}^{f s \times r}, X \in \mathbb{R}^{3 \times r}}\|\operatorname{vec}(Y-M X-N Z)\|_{P \otimes Q_{y y}}^{2}
$$

Its system of normal equations is given as

$$
\begin{aligned}
& S_{1} \cdot\left(\begin{array}{c}
\operatorname{vec}\left(\hat{X}_{\mathrm{uc}}\right) \\
\operatorname{vec}\left(\hat{Z}_{\mathrm{uc}}\right)
\end{array}\right)=\left[\begin{array}{c}
P^{-1} \otimes M^{T} Q_{y y}^{-1} \\
P^{-1} \otimes N^{T} Q_{y y}^{-1}
\end{array}\right] \operatorname{vec}(Y) \\
& S_{1}=\left[\begin{array}{cc}
P^{-1} \otimes M^{T} Q_{y y}^{-1} M & P^{-1} \otimes M^{T} Q_{y y}^{-1} N \\
P^{-1} \otimes N^{T} Q_{y y}^{-1} M & P^{-1} \otimes N^{T} Q_{y y}^{-1} N
\end{array}\right]
\end{aligned}
$$

from which the unconstrained least-squares float solution follows as

$$
\begin{aligned}
& \hat{X}_{\mathrm{uc}}=\left[\bar{M}^{T} Q_{y y}^{-1} \bar{M}\right]^{-1} \bar{M}^{T} Q_{y y}^{-1} Y \\
& \hat{Z}_{\mathrm{uc}}=\left[N^{T} Q_{y y}^{-1} N\right]^{-1} N^{T} Q_{y y}^{-1}\left[Y-M \hat{X}_{\mathrm{uc}}\right]
\end{aligned}
$$

with $\bar{M}=\left[I-N N^{-}\right] M$ and $N^{-}=\left[N^{T} Q_{y y}^{-1} N\right]^{-1} N^{T} Q_{y y}^{-1}$. The v-c matrices that characterize the dispersion of $\hat{X}_{\mathrm{uc}}$ and $\hat{Z}_{\mathrm{uc}}$ follow from applying the variance propagation law to (14) as

$$
\begin{aligned}
Q_{\hat{X}_{\mathrm{uc}} \hat{X}_{\mathrm{uc}}} & =P \otimes\left[\bar{M}^{T} Q_{y y}^{-1} \bar{M}\right]^{-1} \\
Q_{\hat{Z}_{\mathrm{uc}} \hat{X}_{\mathrm{uc}}} & =-P \otimes\left[N^{-} M\left[\bar{M}^{T} Q_{y y}^{-1} \bar{M}\right]^{-1}\right]=Q_{\hat{X}_{\mathrm{uc}} \hat{Z}_{\mathrm{uc}}} \\
Q_{\hat{Z}_{\mathrm{uc}} \hat{Z}_{\mathrm{uc}}} & =P \otimes\left[\bar{N}^{T} Q_{y y}^{-1} \bar{N}\right]^{-1}
\end{aligned}
$$

with $\bar{N}=\left[I-M M^{-}\right] N$ and $M^{-}=\left[M^{T} Q_{y y}^{-1} M\right]^{-1} M^{T} Q_{y y}^{-1}$. Note that expressions (14) and (15) are independent of the actual antenna placement on the platform, since no geometrical information is exploited in the formulation of the UC-AM. 


\section{The Affine-Constrained Float Solution}

Without the integer constraints $Z \in \mathbb{Z}^{f s \times r}$, the minimization problem (9) results in the affine-constrained float solution

$$
\left\{\begin{array}{l}
\hat{Z}_{\mathrm{ac}} \\
\hat{R}_{\mathrm{ac}}
\end{array}\right\}=\arg \min _{Z \in \mathbb{R}^{f s \times r}, R \in \mathbb{R}^{3 \times q}}\|\operatorname{vec}(Y-M R B-N Z)\|_{P \otimes Q_{y y}}^{2}
$$

Its system of normal equations is given as

$$
\begin{gathered}
S_{2} \cdot\left(\begin{array}{c}
\operatorname{vec}\left(\hat{R}_{\mathrm{ac}}\right) \\
\operatorname{vec}\left(\hat{Z}_{\mathrm{ac}}\right)
\end{array}\right)=\left[\begin{array}{c}
B P^{-1} \otimes M^{T} Q_{y y}^{-1} \\
P^{-1} \otimes N^{T} Q_{y y}^{-1}
\end{array}\right] \operatorname{vec}(Y) \\
S_{2}=\left[\begin{array}{cc}
B P^{-1} B^{T} \otimes M^{T} Q_{y y}^{-1} M & B P^{-1} \otimes M^{T} Q_{y y}^{-1} N \\
P^{-1} B^{T} \otimes N^{T} Q_{y y}^{-1} M & P^{-1} \otimes N^{T} Q_{y y}^{-1} N
\end{array}\right]
\end{gathered}
$$

from which the affine-constrained least-squares float solution follows as

$$
\begin{aligned}
\hat{R}_{\mathrm{ac}} & =\left[\bar{M}^{T} Q_{y y}^{-1} \bar{M}\right]^{-1} \bar{M}^{T} Q_{y y}^{-1}[Y] P^{-1} B^{T}\left[B P^{-1} B^{T}\right]^{-1} \\
\hat{Z}_{\mathrm{ac}} & =\left[N^{T} Q_{y y}^{-1} N\right]^{-1} N^{T} Q_{y y}^{-1}\left[Y-M \hat{R}_{\mathrm{ac}} B\right]
\end{aligned}
$$

with v-c matrices

$$
\begin{aligned}
Q_{\hat{R}_{\mathrm{ac}} \hat{\mathrm{R}}_{\mathrm{ac}}} & =\left[B P^{-1} B^{T}\right]^{-1} \otimes\left[\bar{M}^{T} Q_{y y}^{-1} \bar{M}\right]^{-1} \\
Q_{\hat{Z}_{\mathrm{ac}} \hat{R}_{\mathrm{ac}}} & =-B^{T}\left[B P^{-1} B^{T}\right]^{-1} \otimes\left[N^{-} M\left[\bar{M}^{T} Q_{y y}^{-1} \bar{M}\right]^{-1}\right] \\
& =Q_{\hat{\mathrm{R}}_{\mathrm{ac}} \hat{Z}_{\mathrm{ac}}} \\
Q_{\hat{\mathrm{Z}}_{\mathrm{ac}} \hat{\mathrm{Z}}_{\mathrm{ac}}} & =\left[Q_{\hat{Z}_{\mathrm{uc}} \hat{Z}_{\mathrm{uc}}}^{-1}+\right. \\
& \left.P_{S} P^{-1} \otimes N^{T} Q_{y y}^{-1} M\left[M^{T} Q_{y y}^{-1} M\right]^{-1} M^{T} Q_{y y}^{-1} N\right]^{-1}
\end{aligned}
$$

where $P_{S}=I-P^{-1} B^{T}\left(B P^{-1} B^{T}\right)^{-1} B$. The last expression of (19) clearly shows how the affine constraints

improve the precision of the ambiguity float solution, $Q_{\hat{Z}_{\mathrm{ac}} \hat{Z}_{\mathrm{ac}}}<Q_{\hat{Z}_{\mathrm{uc}} \hat{Z}_{\mathrm{uc}}}$, with the latter variance matrix inequality stating that the variance of any linear function of $\hat{Z}_{\mathrm{ac}}$ is smaller than the variance of the same function of $\hat{Z}_{\mathrm{uc}}$. Matrix $P_{S}$ is a projector of rank $r-q$ that projects onto the nullspace of the body frame baseline matrix $B$, see [21]. This matrix reduces to the zero-matrix if the null space is empty, i.e., when $q=r$.

\section{Integer Ambiguity Resolution}

Following [21], the above float solutions can be used to formulate the three different ILS ambiguity solutions as

$$
\begin{aligned}
& \check{Z}_{\mathrm{uc}}=\arg \min _{Z \in \mathbb{Z}^{f s \times r}}\left\|\operatorname{vec}\left(\hat{Z}_{\mathrm{uc}}-Z\right)\right\|_{Q_{\hat{Z}_{\mathrm{uc}} \hat{\mathrm{u}}_{\mathrm{uc}}}^{2}}^{2} \\
& \check{Z}_{\mathrm{ac}}=\arg \min _{Z \in \mathbb{Z}^{f s \times r}}\left\|\operatorname{vec}\left(\hat{Z}_{\mathrm{ac}}-Z\right)\right\|_{Q_{\hat{Z}_{\mathrm{ac}} \hat{z}_{\mathrm{ac}}}}^{2}
\end{aligned}
$$




$$
\begin{aligned}
\check{Z}_{\mathrm{oc}}= & \arg \min _{Z \in \mathbb{Z}^{f s \times r}}\left(\left\|\operatorname{vec}\left(\hat{Z}_{\mathrm{ac}}-Z\right)\right\|_{Q_{\hat{\mathrm{Z}}_{\mathrm{ac}} \hat{Z}_{\mathrm{ac}}}}^{2}\right. \\
& +\min _{R \in \mathbb{O}^{3 \times q}}\left\|\operatorname{vec}\left(\hat{R}_{\mathrm{ac}}(Z)-R\right)\right\|_{Q_{\hat{\mathrm{R}}_{\mathrm{ac}}(Z) \hat{R}_{\mathrm{ac}}(Z)}}^{2}
\end{aligned}
$$

where

$$
\begin{aligned}
& \hat{R}_{\mathrm{ac}}(Z)=\hat{R}_{\mathrm{ac}}-Q_{\hat{R}_{\mathrm{ac}} \hat{Z}_{\mathrm{ac}}} Q_{\hat{Z}_{\mathrm{ac}} \hat{Z}_{\mathrm{ac}}}^{-1}\left(\hat{Z}_{\mathrm{ac}}-Z\right) \\
& Q_{\hat{R}_{\mathrm{ac}}(Z) \hat{R}_{\mathrm{ac}}(Z)}=Q_{\hat{R}_{\mathrm{ac}} \hat{R}_{\mathrm{ac}}}-Q_{\hat{R}_{\mathrm{ac}} \hat{Z}_{\mathrm{ac}}} Q_{\hat{Z}_{\mathrm{ac}} \hat{Z}_{\mathrm{ac}}}^{-1} Q_{\hat{Z}_{\mathrm{ac}} \hat{R}_{\mathrm{ac}}}
\end{aligned}
$$

Since the ambiguity objective functions of both $\check{Z}_{\mathrm{uc}}$ and $\check{Z}_{\mathrm{ac}}$ are quadratic forms in the entries of $Z$, both minimization problems (20) and (21) can be solved by means of the standard LAMBDA method [29]. This is not the case with the ambiguity objective function of (22). Due to the nonlinear orthonormality constraint, the presence of the second term on the right-hand side of (22) makes this ambiguity objective function nonquadratic in $Z$. The solution of this orthonormality-constrained ILS problem (22) is analyzed and tested in [9], [27], and it involves novel search approaches to efficiently extract the integer minimizer. The implementation of these novel strategies has been coined the MC-LAMBDA (Multivariate Constrained-LAMBDA) method. It is shown in [9] that the application of the MC-LAMBDA method is characterized by much higher ambiguity resolution performance as compared to the unconstrained methods. This is due to the presence of the second term on the right-hand side of (22). This term adds complexity to the integer minimization, but also gives $\check{Z}_{\text {oc }}$ a much higher success-rate than $\check{Z}_{\text {uc }}$.

\section{E. Impact of the Affine-Constraints}

We use the Ambiguity Dilution of Precision (ADOP) to get a first impression of the impact of the affineconstraints. The ADOP was introduced in [30] as an easy-to-compute scalar diagnostic to measure the intrinsic model strength for successful ambiguity resolution. The ADOP has been used in [31], [32] and [33] to analyze the benefits to ambiguity resolution of integrating INS with GPS, whereas in [34], the ADOP was used to study the effect the stochastic model has on ambiguity resolution.

The ADOP is defined as the square-root of the ambiguity variance matrix' determinant taken to the power one over the number of DD ambiguities. Thus for the ADOP of the affine-constrained model, we have

$$
\mathrm{ADOP}_{\mathrm{ac}}=\sqrt{\left|Q_{\hat{Z}_{\mathrm{ac}} \hat{Z}_{\mathrm{ac}}}\right|^{\frac{1}{f s r}}}[\mathrm{cycle}]
$$

The ADOP has the important property that it is invariant against the choice of ambiguity parametrization. Since all admissible ambiguity transformations can be shown to have a determinant of one, the ADOP does not change when one changes the definition of the ambiguities. It therefore measures the intrinsic precision of the ambiguities. In fact, it measures the average ambiguity precision, since the ADOP equals the geometric average of the sequential conditional ambiguity standard deviations.

Another important property of the ADOP is its relation to the volume of the ambiguity confidence ellipsoid and the ambiguity search space. The volume of the $f s r$-dimensional ellipsoid $\left[\operatorname{vec}\left(\hat{Z}_{\mathrm{ac}}-Z\right)\right]^{T} Q_{\hat{Z}_{\mathrm{ac}} \hat{Z}_{\mathrm{ac}}}^{-1}\left[\operatorname{vec}\left(\hat{Z}_{\mathrm{ac}}-Z\right)\right] \leq \chi^{2}$ 


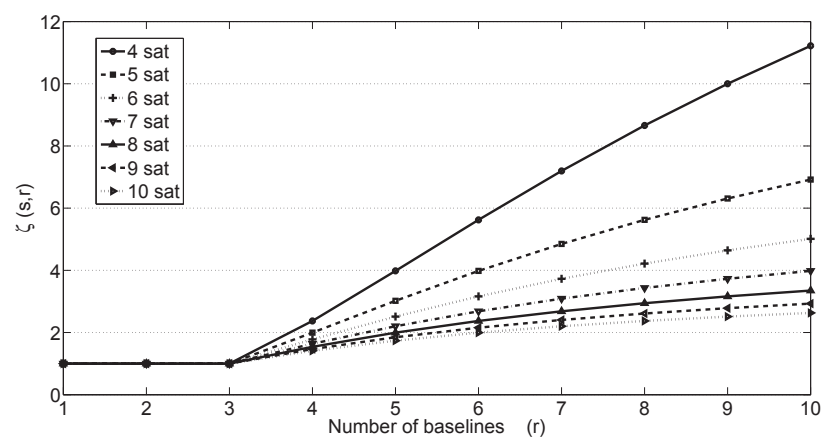

Fig. 2. The gain factor $\zeta$ as function of the number of baselines $r$ and number of tracked satellites $(s+1)$. The variance ratio is assumed to be $\sigma_{p}^{2} / \sigma_{\phi}^{2} \approx 10^{4}$ and $q=\min (3, r)$.

is given as $V_{f s r}=\chi^{f s r} U_{f s r} \mathrm{ADOP}_{\mathrm{ac}}^{f s r}$ where $U_{f s r}$ is the volume of the $f s r$-dimensional unit sphere [35]. Thus if the ADOP gets smaller, the confidence ellipsoid gets smaller and the multivariate PDF of the float ambiguity matrix gets more peaked.

To describe the impact of the affine-constraints, we consider the ratio of the unconstrained ADOP and its affineconstrained counterpart,

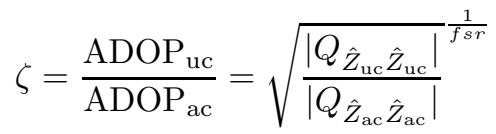

This ratio describes the gain that is experienced due to the affine-constraints. Following the ADOP rule-of-thumb developed in [21], this gain factor $\zeta$ can be expressed analytically, for the single-epoch, single-frequency case, as

$$
\zeta=\left(1+\frac{\sigma_{p}^{2}}{\sigma_{\phi}^{2}}\right)^{\frac{3(r-q)}{2 s r}}
$$

in which $\sigma_{p}^{2} / \sigma_{\phi}^{2}$ denotes the variance ratio of code and phase.

For $r=q$, the ratio $\zeta$ is always equal to one, since the two model formulations UC-AM and AC-AM are then equivalent. When $r>q$, a steep increase of the gain factor can be observed. Assuming the variance ratio to be $\sigma_{p}^{2} / \sigma_{\phi}^{2} \approx 10^{4}$, which is typical for GPS, the gain factor is

$$
\zeta \begin{cases}=1 & \text { for } r=q \leq 3 \\ \approx 10^{\frac{3}{2 s}} & \text { for } r=4, q=3 \\ \approx 10^{\frac{12}{5 s}} & \text { for } r=5, q=3 \\ \approx 10^{\frac{3}{s}} & \text { for } r=6, q=3\end{cases}
$$

Note that

$$
\lim _{r \rightarrow \infty} \zeta=10^{\frac{6}{s}}
$$

which is the maximum gain obtainable when tracking $s+1$ satellites. Figure 2 shows the gain factor $\zeta$ as function of the number of baselines, per number of satellites tracked. For $r>q$, the ambiguity resolution performance of AC-AM is always better than that of UC-AM and it improves with the number of baselines employed. Note that 
this improvement is more significant for weaker models, i.e., when fewer satellites are tracked.

\section{ExPERIMENTAl ANALyses And Results}

In this section the three integer ambiguity estimators (20), (21) and (22) are tested and compared using simulated as well as actual experimental data. Their performances are compared in terms of ambiguity success rates. For the simulation experiments, four different types of success-rate are computed: (1) the actual ILS success-rate based on Monte Carlo simulation, (2) a bootstrapped-based lower-bound $\mathrm{P}_{\mathrm{LB}}$ of the ILS succes-rate, (3) an ADOP-based upper-bound $\mathrm{P}_{\mathrm{UB}}$, and (4) an ADOP-based approximation to the ILS success rate, $\mathrm{P}_{\mathrm{App}}$. The bootstrapped-based lower bound is given as [36],

$$
\mathrm{P}_{\mathrm{LB}}=\prod_{i=1}^{f s r}\left(2 \Phi\left(\frac{1}{2 \sigma_{i \mid I}}\right)-1\right)
$$

with $\Phi(x)=\int_{-\infty}^{x} \frac{1}{\sqrt{2 \pi}} \exp -\frac{1}{2} v^{2} d v$ and $\sigma_{i \mid I}(i=1, \ldots, f s r)$ the sequential conditional standard deviations of the decorrelated ambiguities. The ADOP-based approximation and the ADOP-based upper-bound [37] are given as

$$
\mathrm{P}_{\mathrm{App}} \approx\left(2 \Phi\left(\frac{1}{2 A D O P}\right)-1\right)^{f s r}
$$

and

$$
\mathrm{P}_{\mathrm{UB}}=\mathrm{P}\left(\chi^{2}(f s r, 0) \leq \frac{C_{f s r}}{A D O P^{2}}\right)
$$

where $\chi^{2}(f s r, 0)$ denotes the central Chi-square distribution with $f s r$ degrees of freedom and $C_{f s r}=$ $\left(\frac{f s r}{2} \Gamma\left(\frac{f s r}{2}\right)\right)^{\frac{2}{f s r}} / \pi$, with $\Gamma$ the gamma-function.

For all the experiments, the success-rate analyses are done for the single-epoch, single-frequency case, as this is considered the most challenging scenario. The dependence on model strength is analyzed by varying the number of tracked satellites, the measurement noise and the number of baselines employed.

\section{A. Simulated antenna array}

Simulated data are useful to test the proposed models in an error-controlled environment. The simulation set-up is described in Table I. With the geometry of the GNSS satellites visualized in the Skyplot of Figure 3, several scenarios are simulated with different DD code observation noise $\left(\sigma_{p}=60 / 30 / 10 \mathrm{~cm}\right)$ and DD carrier phase observation noise $\left(\sigma_{\phi}=12 / 6 \mathrm{~mm}\right)$. The data are generated for three to five baselines according to model (1).

As discussed in the previous section, the body frame baseline matrix $B$ plays an important role in the estimation process when employing both the OC-AM and the AC-AM. The consequences are twofold. First, when the number of baselines is higher than their span, the ambiguity success-rates of (20) and (21) differ. In such cases the ACAM provides a more precise ambiguity float solution than the one obtained in the UC-AM approach, which in turn increases the probability of correct integer estimation. Second, ambiguity resolution is also affected by the chosen antenna-array geometry. Poorer baseline configurations yield poorer float solutions, thereby reducing the probability of correct integer estimation. Examples of poor baseline geometries are configurations of baselines with 
TABLE I

SIMULATION SET UP.

\begin{tabular}{cl}
\hline Date and time & 22 Jan 2008 00:00 \\
Location & Lat: $50^{\circ}$, Long: $3^{\circ}$ \\
GPS week & 439 \\
Frequency & L1 \\
\hline Number of Satellite (PRNs) & PDOP \\
$5(8,10,15,26,28)$ & 4.192 \\
$6(8,10,15,26,28,18)$ & 2.142 \\
$7(8,10,15,26,28,18,9)$ & 1.917 \\
$8(8,10,15,26,28,18,9,21)$ & 1.811 \\
\hline DD code noise $\sigma_{p}[\mathrm{~cm}]$ & $60 / 30 / 10$ \\
DD phase noise $\sigma_{\phi}[\mathrm{mm}]$ & $12 / 6$ \\
Wavelength & $\lambda_{f}=0.19029 \mathrm{~m}$ \\
\hline
\end{tabular}

small angular separations. To analyse the impact of the antenna-array geometry, we show results for two different baseline matrices, one having a good geometry, $B_{1}$, and one having a much poorer geometry, $B_{2}$ :

$$
\begin{gathered}
B_{1}=\left[\begin{array}{ccccc}
2 & 1 & 1 & -1 & -2 \\
0 & 1 & 0 & -1 & 2 \\
0 & 0 & 2 & -1 & 2
\end{array}\right] \quad[m] \\
B_{2}=\left[\begin{array}{ccccc}
2 & 1.9 & 1.9 & 1.8 & 1.8 \\
0 & 0.1 & -0.1 & 0 & 0.1 \\
0 & 0 & 0.1 & 0.1 & 0.1
\end{array}\right] \quad[m]
\end{gathered}
$$

We start with $B_{1}$. Next to the five-baseline case, we also consider the three- and four-baseline configurations. They are obtained by taking the first three or four columns of $B_{1}$, respectively.

Figures 4 - 5 visualize the single-epoch, single-frequency success-rates obtained as function of the number of baselines, the noise levels and the number of tracked satellites, for each of the three integer ambiguity estimators $\check{Z}_{\text {uc }}$ (cf. 20), $\check{Z}_{\text {ac }}$ (cf. 21) and $\check{Z}_{\text {oc }}$ (cf. 22). It is clear that the orthonormality-constrained model outperforms the other two models. It achieves a one hundred per cent success-rate in each of the simulated data sets. But note that the AC-AM success-rates increase considerably when passing from three to four and to five-baseline configurations, respectively. Noticeably, it even achieves a one hundred per cent success-rate in one of the five-satellite scenarios. This clearly demonstrates the advantages of the proposed low-complexity affine-constrained GNSS attitude model. Also note that in most of the data sets the bounds and the approximation show a fairly good match with the UC-AM and the AC-AM ILS success rates. This is particularly true for the bootstrapped-based lower bound. However, this is not true anymore, for the success-rates of the MC-LAMBDA method, since the used bounds do not capture all the properties of the orthonormality-constrained attitude model.

Table II compares the single-epoch, single-frequency success-rates for the same scenarios described afore and one with a DD phase observation noise set to $12 \mathrm{~mm}$. The performance of the AC-AM estimator are affected somewhat, 
with lower success rates, whereas the OC-AM success rates remain unchanged (100\% fixed epochs in all the cases simulated, not reported in the table). The success rate differences between same scenarios with different carrier phase noise increase for weaker models, i.e., lower number of satellites and lower number of baselines employed. The affine-constrained method still achieves a $99 \%$ success-rate in one of the five-satellite scenarios.

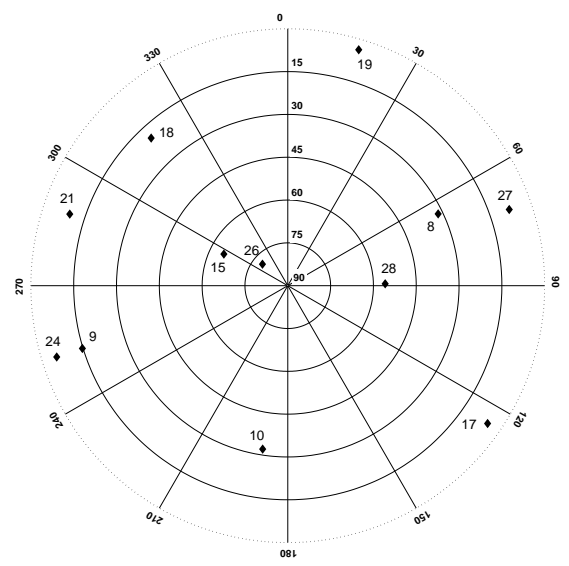

Fig. 3. Skyplot as seen from $50^{\circ}$ latitude, $3^{\circ}$ longitude, on 22 January 2008, 00:00.

The second aspect analyzed is how ambiguity resolution is affected by the geometry of the antenna-array. Compare Figures 6 - 7 for $B_{2}$ with Figures 4 - 5 for $B_{1}$. First, it is observed that the MC-LAMBDA method is relatively insensitive to bad geometrical distributions by virtue of a very strong underlying model. Also the LAMBDA method applied to the UC-AM does not show any variation, since it applies to a model that does not depend on the geometry of the antenna-array. Instead, the LAMBDA method applied to the AC-AM shows a significant dependence on this geometry. Poorer configurations, i.e., smaller angular separations, provide worse results. For example, the fourbaseline scenario with five satellites tracked and $15 \mathrm{~mm}$ code noise shows a largely reduced success rate when using configuration $B_{2}(7 \%)$, compared to the $61 \%$ marked with configuration $B_{1}$. It is thus important to carefully consider the antenna placement onboard the given platform. One should always aim for high angular separations. This would not only improve ambiguity 

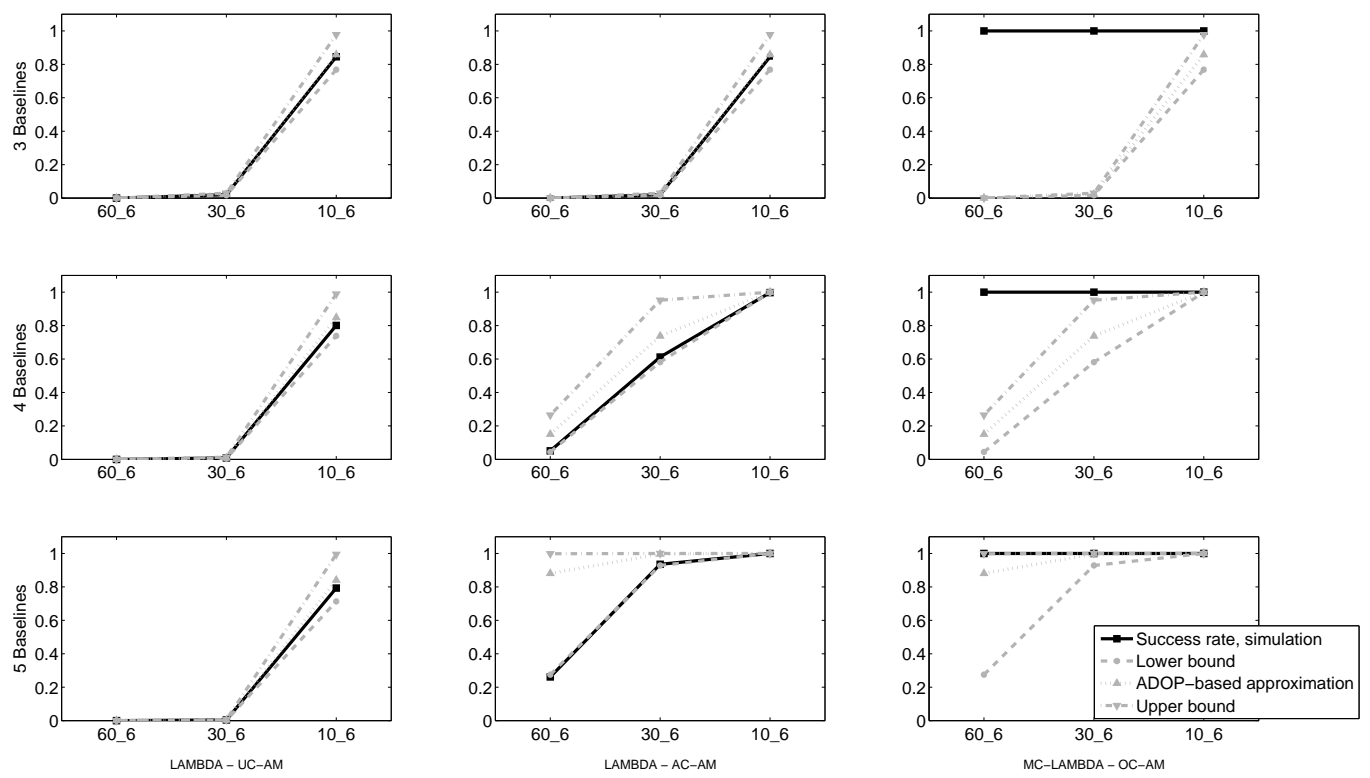

(a) 5 satellites.
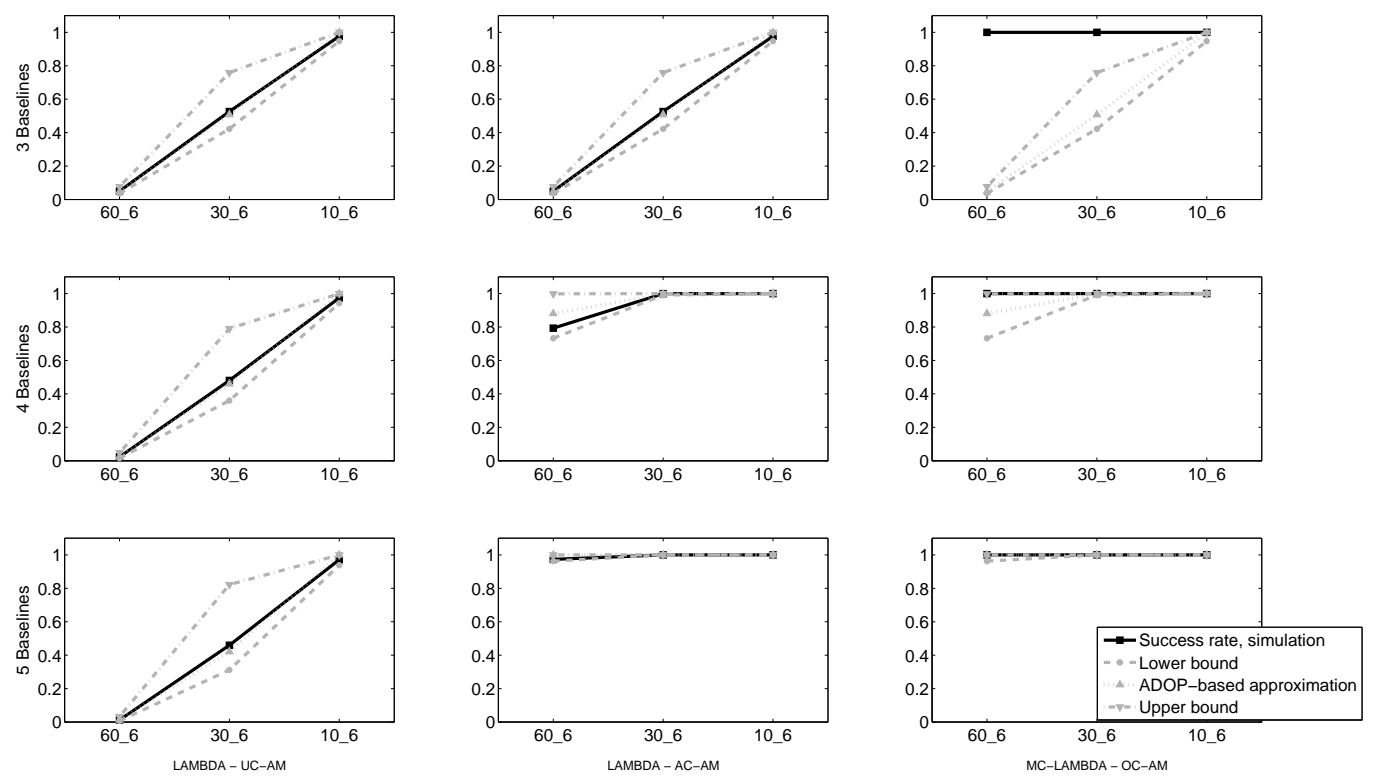

(b) 6 satellites.

Fig. 4. Success rate as function of the DD noise level combination (indicated along the horizontal axis, first two digits denoting the DD pseudorange observation noise in $\mathrm{cm}$, and the following digits denoting the DD carrier phase noise level in mm) and number of baselines employed, for each of the approaches analyzed. Body frame baseline matrix $B_{1} ; 5$ and 6 satellites. 

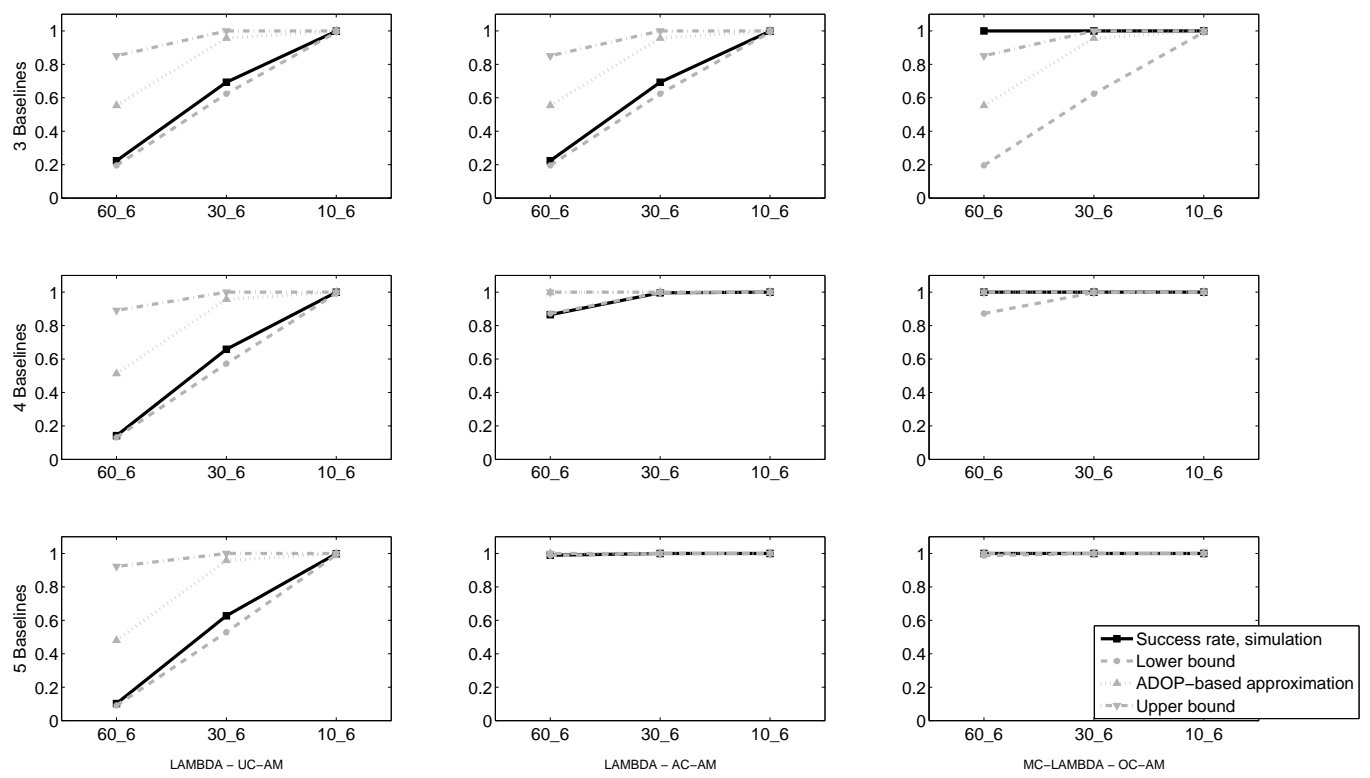

(a) 7 satellites.
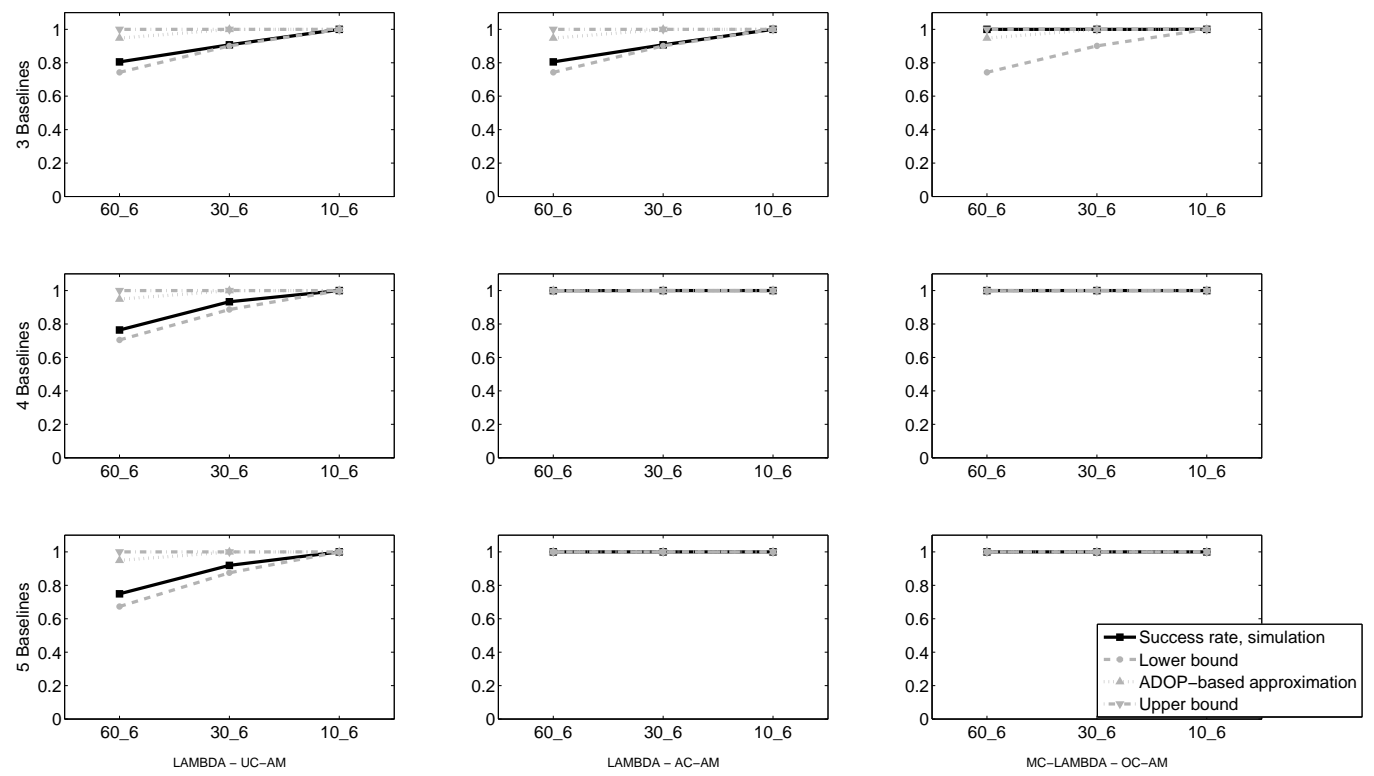

(b) 8 satellites.

Fig. 5. Success rate as function of the DD noise level combination (indicated along the horizontal axis, first two digits denoting the DD pseudorange observation noise in $\mathrm{cm}$, and the following digits denoting the DD carrier phase observation noise level in mm) and number of baselines employed, for each of the approaches analyzed. Body frame baseline matrix $B_{1} ; 7$ and 8 satellites. 

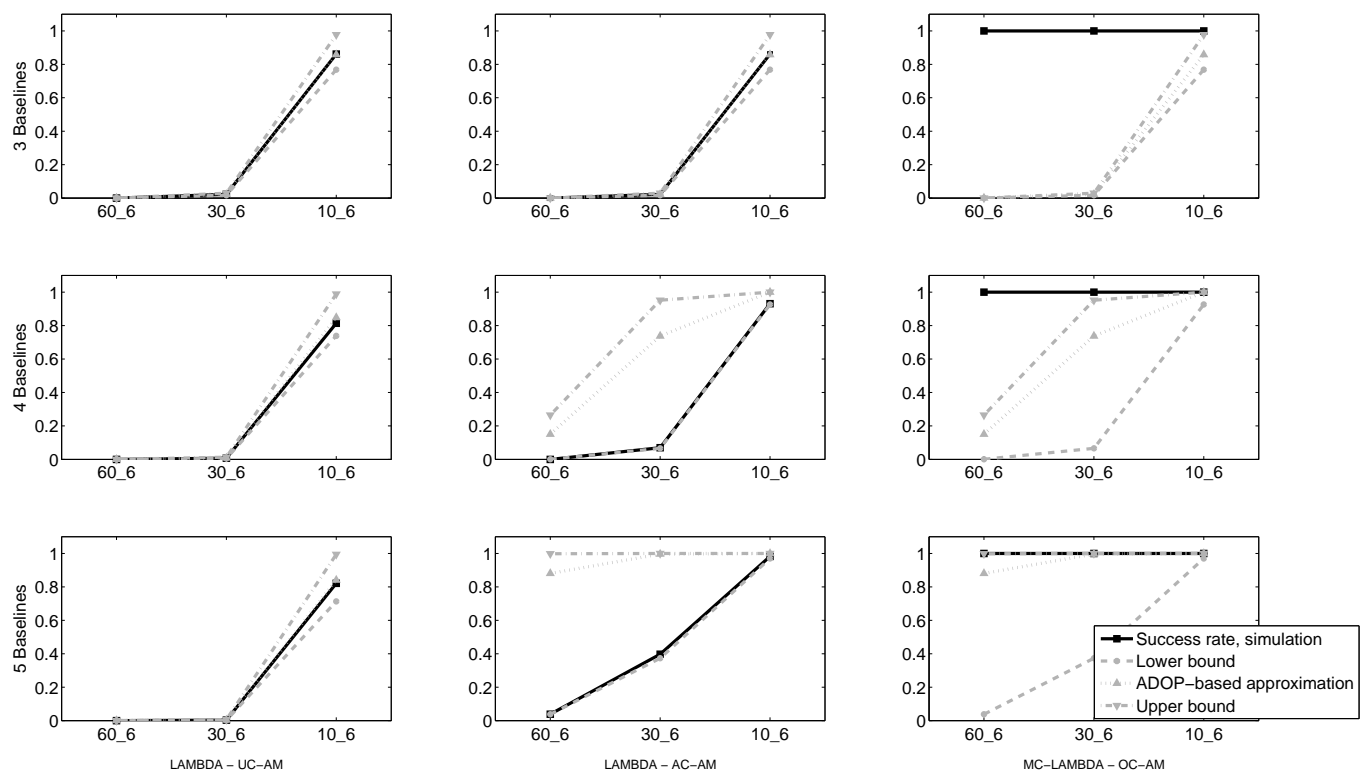

(a) 5 satellites.
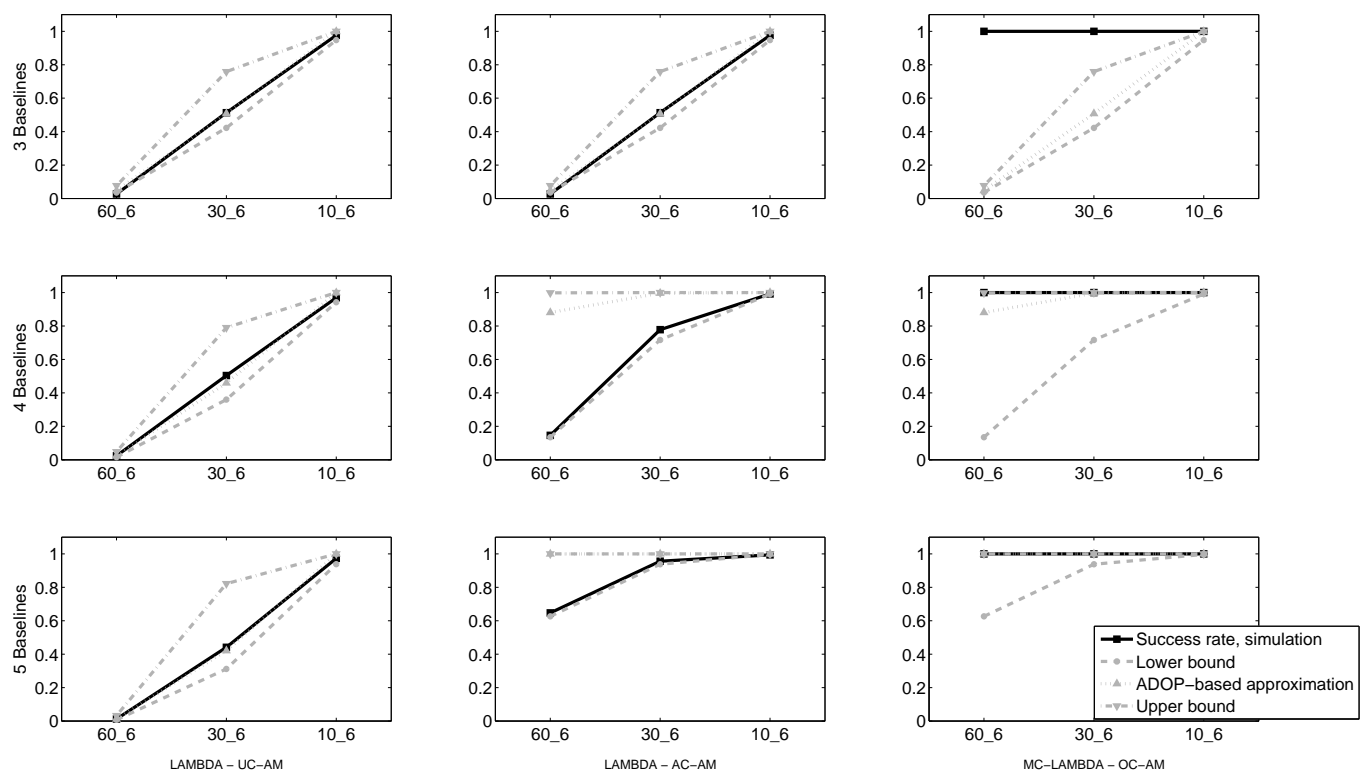

(b) 6 satellites.

Fig. 6. Success rate as function of the DD noise level combination (indicated along the horizontal axis, first two digits denoting the DD pseudorange observation noise in $\mathrm{cm}$, and the following digits denoting the DD carrier phase observation noise level in mm) and number of baselines employed, for each of the approaches analyzed. Body frame baseline matrix $B_{2} ; 5$ and 6 satellites. 

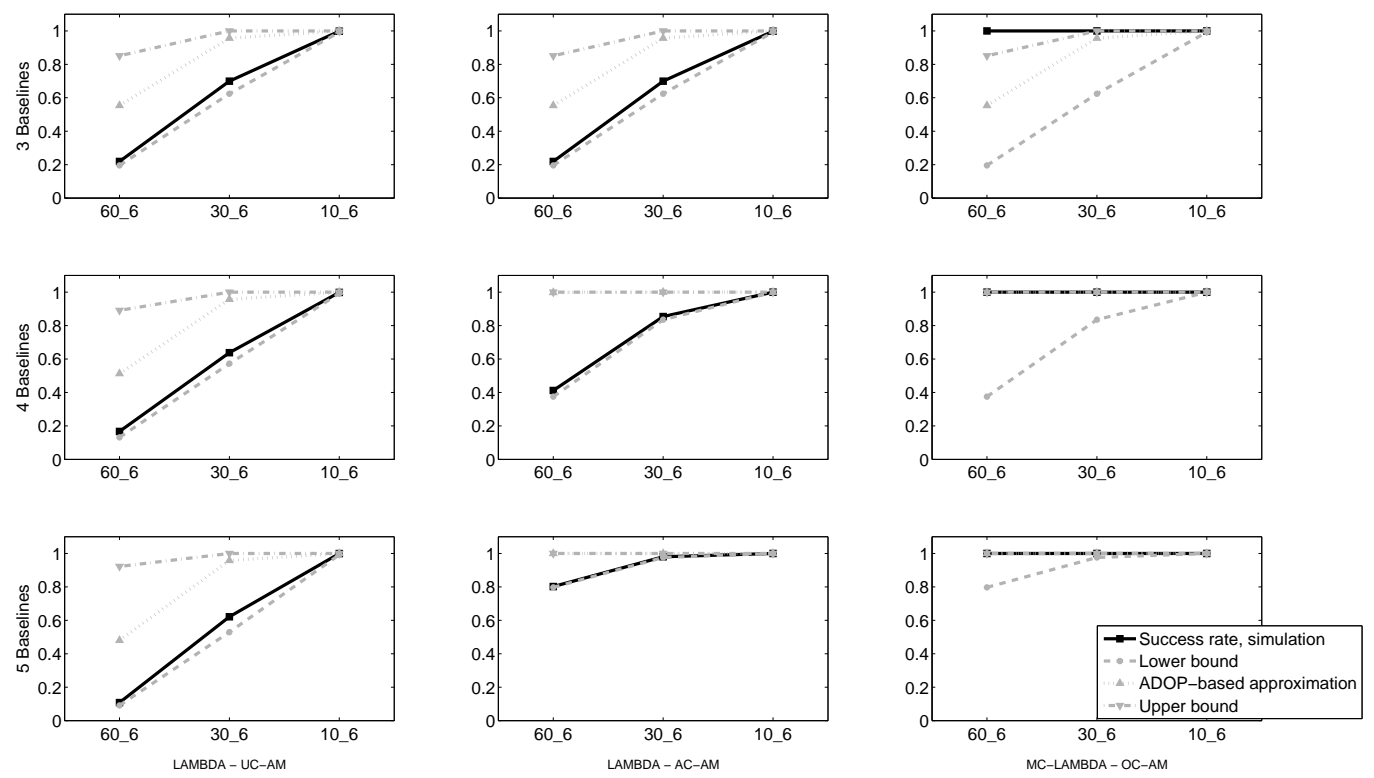

(a) 7 satellites.
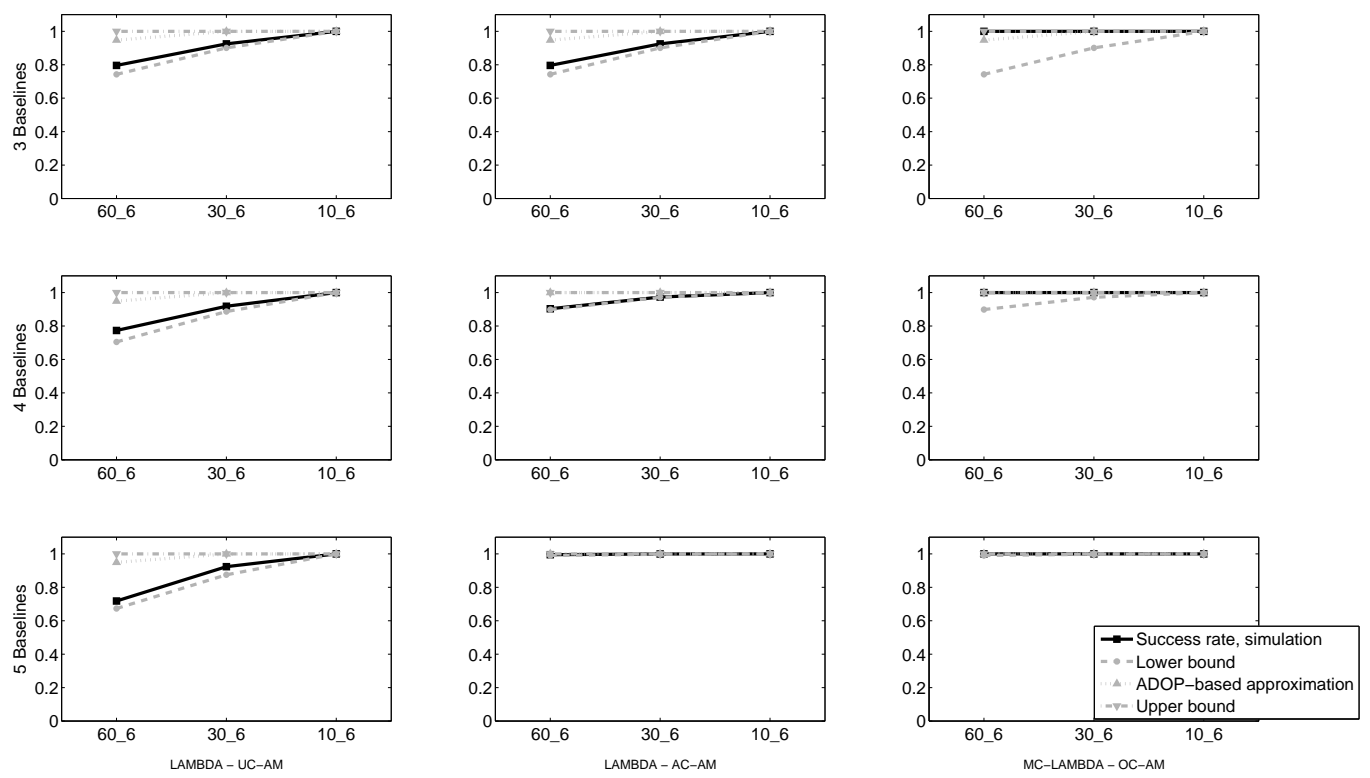

(b) 8 satellites.

Fig. 7. Success rate as function of the DD noise level combination (indicated along the horizontal axis, first two digits denoting the DD pseudorange observation noise in $\mathrm{cm}$, and the following digits denoting the DD carrier phase observation noise level in mm) and number of baselines employed, for each of the approaches analyzed. Body frame baseline matrix $B_{2} ; 7$ and 8 satellites. 
TABLE II

SUCCESS RATE AS FUNCTION OF THE DD NOISE LEVEL COMBINATION, NUMBER OF SATELLITES AND NUMBER OF BASELINES EMPLOYED, FOR THE AC-AM APPROACH AT DIFFERENT NOISE LEVELS. BODY FRAME BASELINE MATRIX $B_{1}$.

\begin{tabular}{cccc|ccc|cccc}
\hline & \multicolumn{3}{c|}{3 baselines } & \multicolumn{3}{c|}{4 baselines } & \multicolumn{3}{c}{5 baselines } \\
\cline { 2 - 10 }$\sigma_{p}[\mathrm{~cm}]$ & 60 & 30 & 10 & 60 & 30 & 10 & 60 & 30 & 10 \\
\hline \multirow{2}{*}{ \# Sat } & \multicolumn{8}{c}{$A C-A M$, DD carrier phase noise set to $6 \mathrm{~mm}$} \\
& \multicolumn{8}{c}{, DD carrier phase noise set to $12 \mathrm{~mm}$} \\
\multirow{2}{*}{5} & 0.00 & 0.02 & 0.85 & 0.05 & 0.61 & 0.99 & 0.26 & 0.94 & 1.00 \\
& 0.00 & 0.01 & 0.59 & 0.02 & 0.30 & 0.96 & 0.21 & 0.87 & 0.99 \\
\hline \multirow{2}{*}{6} & 0.04 & 0.51 & 0.97 & 0.77 & 0.99 & 0.99 & 0.97 & 1.00 & 1.00 \\
& 0.00 & 0.10 & 0.75 & 0.34 & 0.78 & 0.97 & 0.92 & 0.99 & 0.99 \\
\hline \multirow{2}{*}{7} & 0.22 & 0.69 & 0.99 & 0.88 & 0.99 & 1.00 & 0.99 & 1.00 & 1.00 \\
& 0.04 & 0.25 & 0.95 & 0.66 & 0.94 & 0.99 & 0.96 & 1.00 & 1.00 \\
\hline \multirow{2}{*}{8} & 0.79 & 0.92 & 1.00 & 0.99 & 0.99 & 1.00 & 1.00 & 1.00 & 1.00 \\
& 0.26 & 0.69 & 0.99 & 0.93 & 0.99 & 1.00 & 0.99 & 1.00 & 1.00 \\
\hline
\end{tabular}

resolution with the affine-constrained attitude model, but also reduce the computational load for MC-LAMBDA because of the improved float solution.

\section{B. Observed antenna array}

In addition to the simulated experiment of the previous section, we also designed a multi-antenna experiment to test the ambiguity resolution performance of the affine-constrained attitude model with actual GNSS data. Two 'platforms', each carrying five antennas, have been set up by placing ten Trimble receivers (nine R7 models and one SSI model) on top of as many tripods, grouped as depicted in Figures 8a. The receivers $A A$ and $A B$ were connected to Zephyr Geodetic antennas, whereas all the other receivers were connected to Zephyr antennas. The data were collected in the proximity of the Delft University of Technology campus, in Delft, The Netherlands. Each of the receivers was limited to track at most nine GPS satellites during a time span of about one and half hour, for a total of 5400 data-epochs (zero cut-off angle, $1 \mathrm{~Hz}$ sampling), between 08:30 and 10:30 UTC, 6th September 2010. Figure $8 \mathrm{~b}$ reports the number of (common) tracked satellites for the whole set of antennas and corresponding PDOP values, and Figure 8c gives the skyplot for the duration of the experiment. In order to vary the number of available observations, different subsets of the available GNSS satellites have been used, as summarized in Table III. Table IV reports the single-epoch, single-frequency success-rates per number of baselines and per number of tracked satellites. The number of baselines varies from three to nine. Their body frame coordinates are given by 
TABLE III

STATIC DATA-SET: NUMBER AND PRN OF GNSS SATELLITES USED.

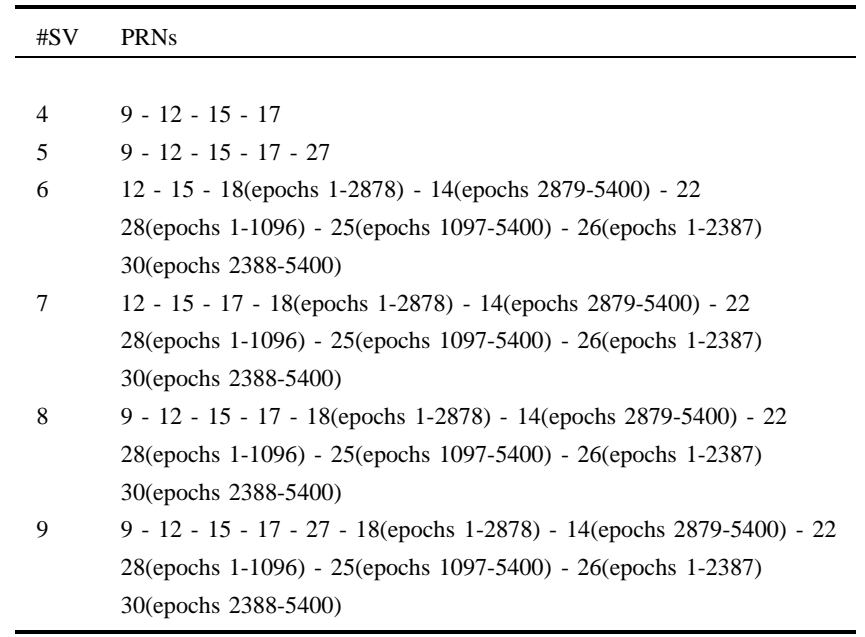

the columns of the baseline matrix $B$ :

$B^{T}=\left[\begin{array}{ccc}1.201 & 0 & 0 \\ 1.790 & 1.249 & 0 \\ 2.475 & 0.186 & 0.088 \\ 0.482 & 0.951 & -0.116 \\ 1.124 & -19.967 & 1.464 \\ 2.406 & 20.080 & 1.448 \\ 3.551 & -20.192 & 1.581 \\ 1.955 & -21.276 & 1.518 \\ 3.195 & -21.012 & 1.609\end{array}\right] \quad[\mathrm{m}]$

For each data set, the LAMBDA method is applied to both the UC-AM and the AC-AM. Results relative to the MC-LAMBDA method are not reported, since the orthonormality-constrained model is already so strong that it always yielded a hundred per cent success rate, in every data set analyzed. For sake of clarity, Figure 9 gives a graphical representation of the results reported in Table IV.

For each of the seven antenna configurations, the number of tracked satellites varies from 4 to 9 . The UC-AM success-rates show a tendency to get smaller as the number of baselines gets larger. This effect is more pronounced when fewer satellites are tracked. This can be explained as follows. If the number of baselines gets larger, the dimension of the ambiguity space gets larger, thus reducing - in the absence of constraints - the probability of correct integer estimation. This reduction gets counteracted however, if more satellites are tracked, which increases the model strength.

The table shows that the AC-AM success-rates are generally significantly larger than the UC-AM success-rates, 


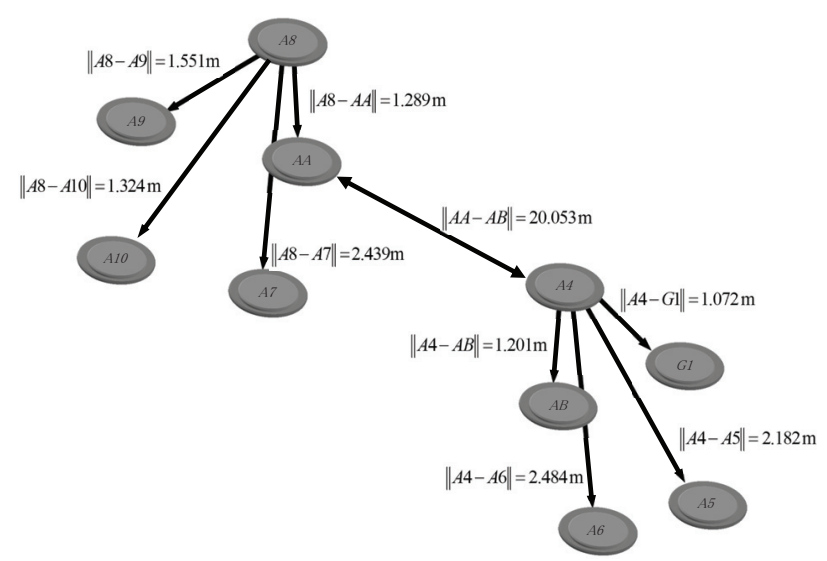

(a) Local baseline geometry.

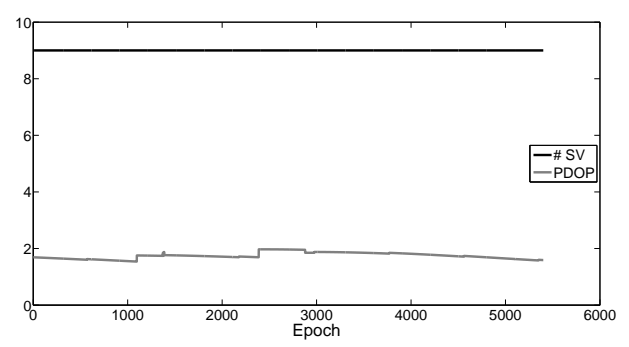

(b) Number of common satellites tracked and PDOP values.

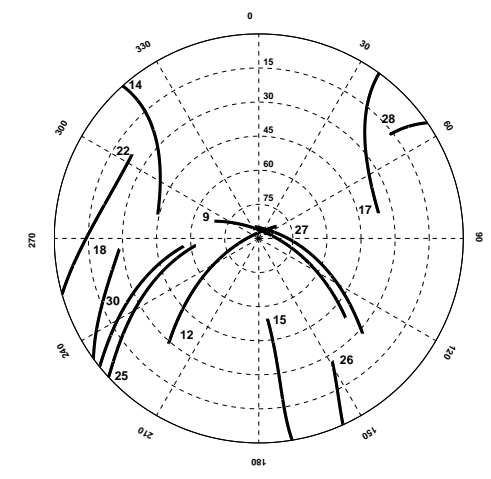

(c) Skyplot.

Fig. 8. Static data-set: test set-up.

especially when the number of satellites is small. The exception is of course the triple baseline case for which $q=r \leq 3$. In that case, UC-AM and AC-AM have the same performance, because of the absence of affineconstraints.

The inclusion of more baselines in the AC-AM steadily increases the success-rate, achieving higher than $99 \%$ success-rates with any number of satellites when ten antennas are employed. This is a noteworthy result: it demonstrates that it is possible to achieve reliable multi-antenna ambiguity resolution employing low-complexity integer search methods, as those of the LAMBDA method. Specifically, when the number of baselines increases, it is possible to achieve reliable results with very few tracked satellites. It is then thus possible to compensate for the 'lack of tracked satellites' by using more antennas in the array. This compensation of a lack of 'external' information by 'local' information confirms the analytical findings of [21].

\section{CONCLuSion}

In this contribution a new affine-constrained approach to the problem of ambiguity resolution for attitude determination applications was tested. Its performance was compared to the performances of the unconstrained 
TABLE IV

STATIC DATA-SET: UNAIDED SINGLE-EPOCH, SINGLE-FREQUENCY SUCCESS-RATES (\%) FOR THE LAMBDA METHOD APPLIED TO UC-AM AND AC-AM, PER NUMBER OF TRACKED SATELLITES AND PER NUMBER OF USED BASELINES.

\begin{tabular}{|c|c|c|c|}
\hline Baseline configurations & \# SV & $\begin{array}{c}\text { LAMBDA } \\
\text { UC-AM }\end{array}$ & $\begin{array}{c}\text { LAMBDA } \\
\text { AC-AM } \\
\end{array}$ \\
\hline \multirow{6}{*}{ A4-AB-A5-A6 } & 4 & 0.1 & 0.1 \\
\hline & 5 & 1.2 & 1.2 \\
\hline & 6 & 10.6 & 10.6 \\
\hline & 7 & 57.5 & 57.5 \\
\hline & 8 & 93.9 & 93.9 \\
\hline & 9 & 99.8 & 99.8 \\
\hline \multirow{6}{*}{ A4-AB-A5-A6-G1 } & 4 & 0 & 0.6 \\
\hline & 5 & 0.1 & 18.9 \\
\hline & 6 & 4.2 & 75.9 \\
\hline & 7 & 58.0 & 99.3 \\
\hline & 8 & 94.3 & 100 \\
\hline & 9 & 99.9 & 100 \\
\hline \multirow{6}{*}{ A4-AB-A5-A6-G1-8 } & 4 & 0 & 35.5 \\
\hline & 5 & 0 & 80.9 \\
\hline & 6 & 2.3 & 97.8 \\
\hline & 7 & 53.7 & 99.3 \\
\hline & 8 & 94.0 & 100 \\
\hline & 9 & 99.9 & 100 \\
\hline \multirow{6}{*}{ A4-AB-A5-A6-G1-A8-AA } & 4 & 0 & 82.2 \\
\hline & 5 & 0 & 95.4 \\
\hline & 6 & 1.5 & 99.0 \\
\hline & 7 & 50.4 & 99.9 \\
\hline & 8 & 93.0 & 100 \\
\hline & 9 & 99.9 & 100 \\
\hline \multirow{6}{*}{ A4-AB-A5-A6-G1-A8-AA-A7 } & 4 & 0 & 89.9 \\
\hline & 5 & 0 & 97.1 \\
\hline & 6 & 1.1 & 99.4 \\
\hline & 7 & 48.9 & 99.9 \\
\hline & 8 & 93.3 & 100 \\
\hline & 9 & 99.1 & 100 \\
\hline \multirow{6}{*}{ A4-AB-A5-A6-G1-A8-AA-A7-A9 } & 4 & 0 & 98.0 \\
\hline & 5 & 0 & 98.8 \\
\hline & 6 & 0.9 & 99.7 \\
\hline & 7 & 45.5 & 100 \\
\hline & 8 & 92.7 & 100 \\
\hline & 9 & 99.6 & 100 \\
\hline \multirow{6}{*}{ A4-AB-A5-A6-G1-A8-AA-A7-A9-A10 } & 4 & 0 & 99.1 \\
\hline & 5 & 0 & 99.5 \\
\hline & 6 & 0.6 & 99.7 \\
\hline & 7 & 44.2 & 100 \\
\hline & 8 & 92.5 & 100 \\
\hline & 9 & 99.9 & 100 \\
\hline
\end{tabular}

and the orthonormality-constrained GNSS attitude models.

The affine-constrained approach exploits the geometrical information about the antenna configuration in a novel fashion. The unknown real-valued baseline coordinates are parameterized in terms of an affine transformation, thus 


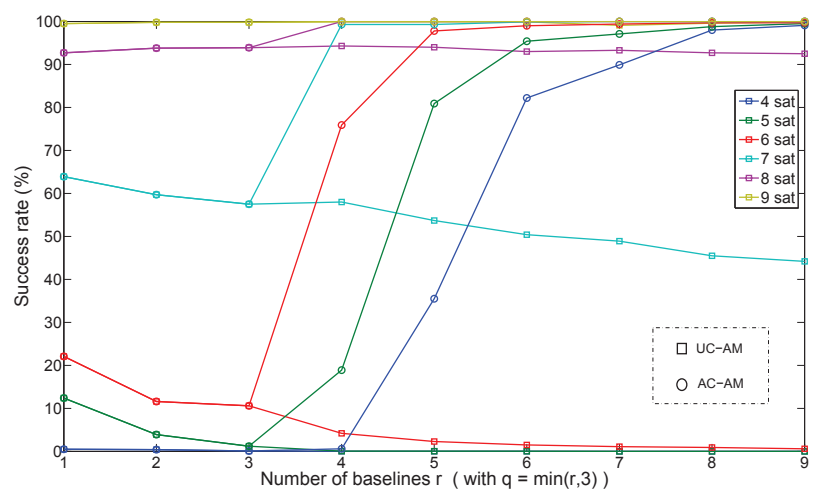

Fig. 9. Static data-set: single-epoch, single-frequency success rate for the LAMBDA method applied to the UC-AM or to the AC-AM, per number of satellites and number of baselines included in the model.

leading to a dimensional reduction in the unknown set of variables whenever the number of baselines exceeds their span. This approach avoids the computational complexity of the nonlinear orthonormality-constrained approaches, since only linear constraints are used. Hence, standard methods of ambiguity resolution, like the LAMBDA method, can be directly applied.

The strength of the affine-constrained attitude model was tested through simulations and actual experimental data. It was shown that very high, single-frequency, single-epoch success-rates can be achieved.

The strength of the model depends on the number of baselines employed, the number of satellites tracked and the observational noise levels. For the affine-constrained attitude model, it was found that for a DD code-phase noise of $60 \mathrm{~cm}-12 \mathrm{~mm}$, single-epoch successful ambiguity resolution is possible when tracking seven satellites with six antennas, while for $30 \mathrm{~cm}-6 \mathrm{~mm}$ this already becomes possible with six satellites and 5 antennas. Hence, the affine-constrained approach allows one to achieve a reliable ambiguity resolution performance by compensating with additional local antennas/receivers any weakness of the given observational model, like e.g., small number of tracked satellites or large measurement noise.

Also, various easy-to-compute success-rate bounds were computed and evaluated. The bootstrapped-based bound was found to be a sharp and easy-to-compute lower bound for the AC-AM success rate.

\section{ACKNOWLEDGMENT}

P.J.G. Teunissen is the recipient of an Australian Research Council Federation Fellowship (project number FF0883188): this support is greatly acknowledged. This work has been carried out in the context of the Australian Space Research Program GARADA project on SAR Formation Flying. The authors would like to thank P.J. Buist, from the TU Delft, for providing the static data-set.

\section{REFERENCES}

[1] P. Axelrad and L. Ward, "On-Orbit GPS Based Attitude and Antenna Baseline Estimation," Proceedings of ION-NTM, San Diego, CA, US, pp. 441-450, 1994. 
[2] I. Y. Bar-Itzhack, P. Montgomery, and J. Garrick, "Algorithm for Attitude Determination using Global Positioning System," Journal of Guidance, Control and Dynamics, vol. 21, no. 6, pp. 846-852, 1998.

[3] R. A. Brown, "Instantaneous GPS Attitude Determination," Proceedings of IEEE Position Location and Navigation Symposium, PLANS 92, US, pp. 113-120, 1992.

[4] C. E. Cohen, "Attitude Determination Using GPS," Ph.D. Thesis, Stanford University, Palo Alto, CA, USA, 1992.

[5] J. L. Crassidis, F. L. Markley, and E. G. Lightsey, "A New Algorithm for Attitude Determination Using Global Positioning System Signals," AIAA Journal of Guidance, Control, and Dynamics, vol. 20, no. 5, pp. 891-896, 1997.

[6] L. Dai, K. V. Ling, and N. Nagarajan, "Real-time Attitude Determination for Microsatellite by LAMBDA Method Combined with Kalman Filtering," Proceedings of 22nd AIAA International Communications Satellite Systems Conference and Exhibit 2004 (ICSSC), Monterey, California, US, 2004.

[7] H. J. Euler, "Attitude determination: Exploiting All Information for Optimal Ambiguity Resolution,” Proceedings of ION GPS, pp. 1751$1757,1995$.

[8] G. Giorgi and P. J. Buist, "Single-epoch, Single Frequency, Standalone Full Attitude Determination: Experimental Results." Proceedings of 4th ESA Workshop on Satellite Navigation User Equipment Technologies, NAVITEC08, ESA-ESTEC, The Netherlands, 2008.

[9] G. Giorgi, P. J. G. Teunissen, S. Verhagen, and P. J. Buist, "Instantaneous Ambiguity Resolution in GNSS-based Attitude Determination Applications: the MC-LAMBDA method," Journal of Guidance, Control, and Dynamics, vol. 35, no. 1, pp. 51-67, 2011.

[10] A. Hauschild and O. Montenbruck, "GPS-Based Attitude Determination for Microsatellite," Proceedings of ION GNSS, Forth Worth, TX, USA, pp. 2424-2434, 2007.

[11] L. S. D. Kim and R. B. Langley, "GPS Ambiguity Resolution and Validation: Methodologies, Trends and Issues," 7th GNSS Workshop International Symposium on GPS/GNSS, Seul, Korea, 2000.

[12] L. V. Kuylen, F. Boon, and A. Simsky, “Attitude Determination Methods Used in the PolarRx2@ Multi-antenna GPS Receiver,” Proceedings of ION GPS, Long Beach, CA, US, pp. 125-135, 2005.

[13] Y. Li, K. Zhang, C. Roberts, and M. Murata, "On-the-fly GPS-based Attitude Determination Using Single- and Double-Differenced Carrier Phase Measurements," GPS Solutions, vol. 8, no. 2, pp. 93-102, 2004.

[14] J. Madsen and E. G. Lightsey, "Robust Spacecraft Attitude Determination Using Global Positioning System Receivers," Journal of Spacecraft and Rockets, vol. 41, no. 4, pp. 635-643, 2004.

[15] R. Monikes, J. Wendel, and G. F. Trommer, "A Modified LAMBDA Method for Ambiguity Resolution in the Presence of Position Domain Constraints," Proceedings of ION GPS, Long Beach, CA, US, pp. 18-24, 2005.

[16] M. L. Psiaki, "Batch Algorithm for Global-Positioning-System Attitude Determination and Integer Ambiguity Resolution," Journal of Guidance, Control, and Dynamics, vol. 29, no. 5, pp. 1071-1079, 2006.

[17] J. Schleppe, "A Real-Time Attitude System Using a Quaternion Parameterization," Proceedings of KIS97, pp. 395-408, 1997.

[18] S. Verhagen, "The GNSS Integer Ambiguities: Estimation and Validation," PhD dissertation, Delft University of Technology, Delft, The Netherlands, 2004.

[19] P. J. Buist, "The Baseline Constrained LAMBDA Method for Single Epoch, Single Frequency Attitude Determination Applications," Proceedings of ION GPS, Fort Worth, TX, US, pp. 2962-2973, 2007.

[20] P. J. G. Teunissen, "A General Multivariate Formulation of the Multi-Antenna GNSS Attitude Determination Problem," Artificial Satellites, vol. 42, no. 2, pp. 97-111, 2007.

[21] — - "The Affine Constrained GNSS Attitude Model and its Multivariate Integer Least-Squares Solution," Journal of Geodesy. DOI: 10.1007/s00190-011-0538-z., 2011.

[22] P. J. G. Teunissen and A. Kleusberg, “GPS for Geodesy,” Springer, Berlin Heidelberg New York, 1998.

[23] J. R. Magnus and H. Neudecker, Matrix Differential Calculus with Applications in Statistics and Econometrics. John Wiley and Sons, 1995.

[24] D. A. Harville, Matrix Algebra From A Statistician's Perspective. Springer Verlag, New York, Berlin, Heidelberg, 1997.

[25] M. D. Shuster, "The Quest for Better Attitudes," The Journal of the Astronautical Sciences, vol. 54, no. 3-4, pp. 657-683, 2006.

[26] G. Giorgi, "GNSS Carrier Phase-based Attitude Determination. Estimation and Applications," PhD dissertation, Delft University of Technology, Delft, The Netherlands, 2011. 
[27] G. Giorgi, P. J. G. Teunissen, S. Verhagen, and P. J. Buist, "Testing a New Multivariate GNSS Carrier Phase Attitude Determination Method for Remote Sensing Platforms," Advances in Space Research, vol. 46, no. 2, pp. 118-129, 2010.

[28] P. J. G. Teunissen, "Integer Least-Squares Theory for the GNSS Compass," Journal of Geodesy, vol. 84, pp. 433-447, 2010.

[29] — - "The Least-Squares Ambiguity Decorrelation Adjustment: a Method for Fast GPS Integer Ambiguity Estimation," Journal of Geodesy, vol. 70, no. 1-2, pp. 65-82, 1995.

[30] — _ "A Canonical Theory for Short GPS Baselines. Part IV: Precision versus Reliability," Journal of Geodesy, vol. 71, no. 9, pp. 513-525, 1997.

[31] J. Skaloud, "Reducing the GPS Ambiguity Search Space by Including Inertial Data," Proceedings of The Institute of Navigations ION GPS-98, pp. 2073-2080, 1998.

[32] B. M. Scherzinger, "Precise Robust Positioning with Inertial/GPS RTK," Proceedings of The Institute of Navigations ION GPS-2000, pp. $155-162,2000$.

[33] — - "Inertially-Aided RTK Position Measurement," Proceedings of the International Symposium on Kinematic Systems in Geodesy, Geomatics and Navigation, Department of Geomatics Engineering, The University of Calgary, pp. 265-272, 2001.

[34] J. Wang, H. K. Lee, Y. J. Lee, T. Musa, and C. Rizos, "Online Stochastic Modelling for Network-Based GPS Real-Time Kinematic Positioning," Journal of Global Positioning Systems, vol. 4, no. 1-2, pp. 113-119, 2005.

[35] P. J. G. Teunissen, P. J. de Jonge, and C. C. J. M. Tiberius, "The Volume of the GPS Ambiguity Search Space and its Relevance for Integer Ambiguity Resolution,” Proc. of ION GPS-1996, Kansas City MO, pp. 889-898, 1996.

[36] P. J. G. Teunissen, "Success Probability of Integer GPS Ambiguity Rounding and Bootstrapping," Journal of Geodesy, vol. 72, no. 10, pp. 606-612, 1998.

[37] — _ "ADOP based Upperbounds for the Bootstrapped and the Least-Squares Ambiguity Success Rates," Artificial Satellites, vol. 35, no. 4, pp. 171-179, 2000.

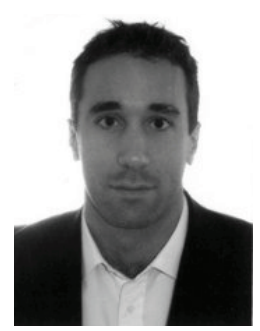

Gabriele Giorgi is a researcher at the Institute for Communications and Navigation, Technische Universität München, in Munich,Germany. He obtained a PhD following his work on Global Navigation Satellite System (GNSS) for aerospace applications from the Delft Institute of Earth Observation and Space Systems (DEOS), Delft University of Technology, in Delft, The Netherlands. His current research focus is on reliable GNSS positioning for aeronautics applications. He has an MSc degree in space engineering from the University of Rome "La Sapienza" and a BSc degree in aerospace engineering from the same university.

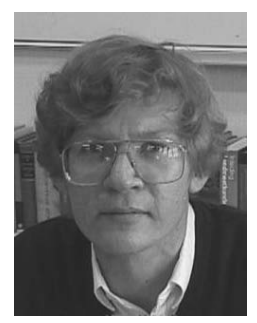

Peter J. G. Teunissen is Federation Fellow of the Australian Research Council (ARC), professor of geodesy and navigation and leader of CUT's Global Navigation Satellite System (GNSS) research lab. His current research focus is on next-generation GNSS for relative navigation and attitude determination in space and air. He is the inventor of the LAMBDA method and has 25 years of GNSS research experience 